\title{
Modelling wave propagation across a series of gaps
}

\author{
Gavin R. Armstrong, ${ }^{* a}$ Annette F. Taylor, ${ }^{a}$ Stephen K. Scott ${ }^{a}$ and Vilmos Gáspár ${ }^{b}$ \\ ${ }^{a}$ Department of Chemistry, University of Leeds, Leeds, UK LS2 9JT. \\ E-mail: G.Armstrong@chem.leeds.ac.uk \\ ${ }^{b}$ Department of Chemistry, University of Debrecen, P. O. Box 74010 Debrecen, Hungary
}

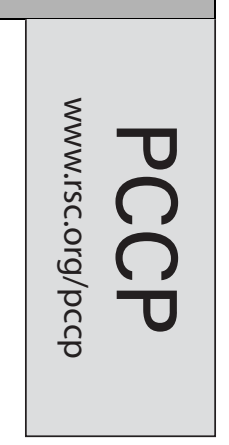

Received 27th April 2004, Accepted 30th July 2004

First published as an Advance Article on the web 18th August 2004

Wave propagation across a series of gaps in a one-dimensional excitable medium is simulated using the Oregonator model of the Belousov-Zhabotinsky reaction. In agreement with recent experiments, we observe features such as the critical gap width $W_{\mathrm{cr}}$, critical spacing between gaps $S_{\mathrm{cr}}$ and frequency transformation of the passage of a train of waves across a gap with width $W \leq W_{\mathrm{cr}}$. The role of activator kinetics in the gap is studied and the effect of excitability (through variation of parameters $f$ and $\varepsilon$ ) on the fraction of waves which successfully cross the domain is determined. We also find that the probability of a wave successfully propagating through the entire domain decreases with increasing number of gaps, and the profile of the activator species is examined for evidence of a "weakening" effect in a multiple gap system.

\section{Introduction}

Excitable media such as the heart muscle or nerve tissue are capable of supporting chemical/electrical waves of activity. The Belousov-Zhabotinsky reaction has been extensively used as a chemical model to investigate generic dynamical features of excitable media. ${ }^{1}$ Current studies focus on the propagation of waves in heterogeneous media, for which the excitability is a function of spatial coordinate, a situation which is more likely to occur in biological systems than one of uniform excitability. ${ }^{2,3}$ Heterogeneity may contribute to wave break-up and spiral formation in the heart, a phenomenon associated with cardiac arrhythmia and ventricular fibrillation. ${ }^{4,5}$ Multiple mechanisms for this dangerous heart condition have been proposed, but a deeper understanding of the effect of heterogeneity on wave propagation is required. ${ }^{6,7}$

Experiments and simulations have demonstrated that waves are capable of propagating across a single gap in the medium i.e. a region of low or no excitability. ${ }^{8-10}$ Passage of the wave is thought to involve passive diffusion of an activator species across the gap and a supercritical threshold of activator is required to initiate activity on the other side of the gap. There exists a critical gap width $\left(W_{\mathrm{cr}}\right)$ for which waves passing across a larger gap will fail, a phenomenon known as wave block. ${ }^{11}$ Moreover, a gap close in width but less than $W_{\text {cr }}$ can act as a frequency transformer, such that only a fraction of waves $f_{n}$ in a wave train will cross the gap, for every wave that enters. Thus we define a firing number $f_{n}=m / n$, where $m$ is the number of waves transmitted across a gap (or an array of gaps) and $n$ is the number of incident waves. ${ }^{12,13}$ This phenomenon has also been observed in experiments on chemical wave propagation through capillary tubes and can be explained by the existence of a refractory period following excitation, i.e. a period of time during which the excitable medium recovers its excitability. ${ }^{14}$ The threshold of activator required to stimulate excitation is much higher during the recovery time.

Recent experiments investigated the passage of a wave across a series of gaps in the excitable domain. ${ }^{8}$ The excitable domain was constructed by using a DeskJet printer to print the reaction catalyst onto a membrane thereby fixing the catalyst in a defined pattern. This pattern had a fixed-frequency wave source and several lanes of catalyst interrupted by gaps. The experiments confirmed the existence of a critical spacing between gaps $S_{\mathrm{cr}}$ for successful propagation of a wave across the entire system. A "weakening" effect was also observed, whereby for systems with $S$ and $W$ close to the critical values, waves failed to propagate farther than seven gaps.

In this paper, we simulate the propagation of waves across a series of gaps in a one-dimensional excitable domain using the two-variable Oregonator model, ${ }^{15}$ which is generally used to model the Belousov-Zhabotinsky reaction. This model also serves as a paradigm for biological excitable media. We determine the fraction of waves successfully propagating across the entire domain and how this depends on the gap width, spacing between gaps and the activator kinetics in the gap. We also examine the concentration profile of the activator species to investigate a possible "weakening" of the wave by the existence of multiple gaps.

\section{Model}

Numerical simulations were performed using the two-variable Oregonator model, based on the kinetic scheme of Field, Körös and Noyes. ${ }^{16}$ We applied the scalings of Tyson and Fife $^{17}$ and assumed diffusion only of the activator species $(u)$ in one spatial dimension $(x)$ only:

$$
\begin{gathered}
\frac{\partial u}{\partial \tau}=\frac{\partial^{2} u}{\partial x^{2}}+\frac{1}{\varepsilon}\left\{u(1-u)-f v \frac{(u-q)}{(u+q)}\right\} \\
\frac{\partial v}{\partial \tau}=u-v
\end{gathered}
$$

where $u$ and $v$ are the dimensionless concentrations of the autocatalyst $\mathrm{HBrO}_{2}$ and oxidized form of the metal catalyst $\mathrm{M}_{\mathrm{ox}}$ respectively and the parameters $\varepsilon, f$ and $q$ can be related to rate constants and initial concentrations. The values of the parameters were chosen to reflect typical experimental conditions: $\varepsilon=0.02-0.07, f=2.4-3.1$ and $q=8 \times 10^{-4}$. The excitability of the medium can be considered to decrease as $\varepsilon$ is increased from 0.02 to 0.07 and as $f$ is increased from 2.4 to 3.1. The equations were integrated using XPPAUT ${ }^{18}$ in a 1-d spatial domain. The integration method used was "cvode" with dimensionless timestep $\mathrm{d} \tau=0.0005$, and tolerance $=1 \times 10^{-7}$. Time was scaled by $\tau=t k_{\mathrm{c}} B$, where $t$ is dimensional time, $k_{\mathrm{c}}$ is a rate constant and $B$ relates to initial malonic acid 
concentration in the Belousov-Zhabotinsky system. The values for $k_{\mathrm{c}}$ and $B$ were $1 \mathrm{M}^{-1} \mathrm{~s}^{-1}$ and $0.17 \mathrm{M}$ respectively. Space was scaled by $x=l /\left(D / k_{\mathrm{c}} B\right)^{1 / 2}$, where $l$ is the dimensional length and $D$ is the diffusion coefficient for the activator species. In aqueous media $D$ is typically $2 \times 10^{-5} \mathrm{~cm}^{2} \mathrm{~s}^{-1}$ but since we are modelling a fixed-catalyst system we assume a reduced diffusion coefficient that was chosen so as to match experimental wave speeds. ${ }^{8}$ Therefore the value of $D$ was chosen to be $5 \times 10^{-6} \mathrm{~cm}^{2} \mathrm{~s}^{-1}$. Note, however, that with the scaling applied the dimensionless value of diffusion coefficient is set exactly to unity. The dimensionless length of the integration domain has been varied between $L=50$ and 100 , corresponding to a dimensional length of between 2.7 and $5.4 \mathrm{~mm}$. The grid spacing applied to calculate the diffusion term at each grid point by the standard three-point-formula (method-of-lines) was fixed $(h=0.25)$ resulting in a onedimensional domain of between 200 and 400 excitable units. No-flux boundary conditions were introduced at the borders of the domain.

The period of initiation was governed by application of a (temporally) periodic function for the concentration of $u$ over the first five grid points with $u_{\max }=0.1$.

Excitable zones (described by eqns. (1) and (2)) were separated by gaps in which:

$$
\begin{gathered}
\frac{\partial u}{\partial \tau}=\frac{\partial^{2} u}{\partial x^{2}}-g(u) \\
\frac{\partial v}{\partial \tau}=0
\end{gathered}
$$

where $g(u)$ is given by $g(u)=u^{2} / \varepsilon$; where $\varepsilon=0.054$. This reflects the most realistic kinetics of the Belousov-Zhabotinsky reaction within the gap i.e. the disproportionation of bromous acid. ${ }^{16} \mathrm{We}$ also investigated zero-order kinetics in the gap, i.e. diffusion but no chemistry $(g(u)=0)$ and first-order kinetics $(g(u)=u / \varepsilon$, where $\varepsilon=0.054)$.
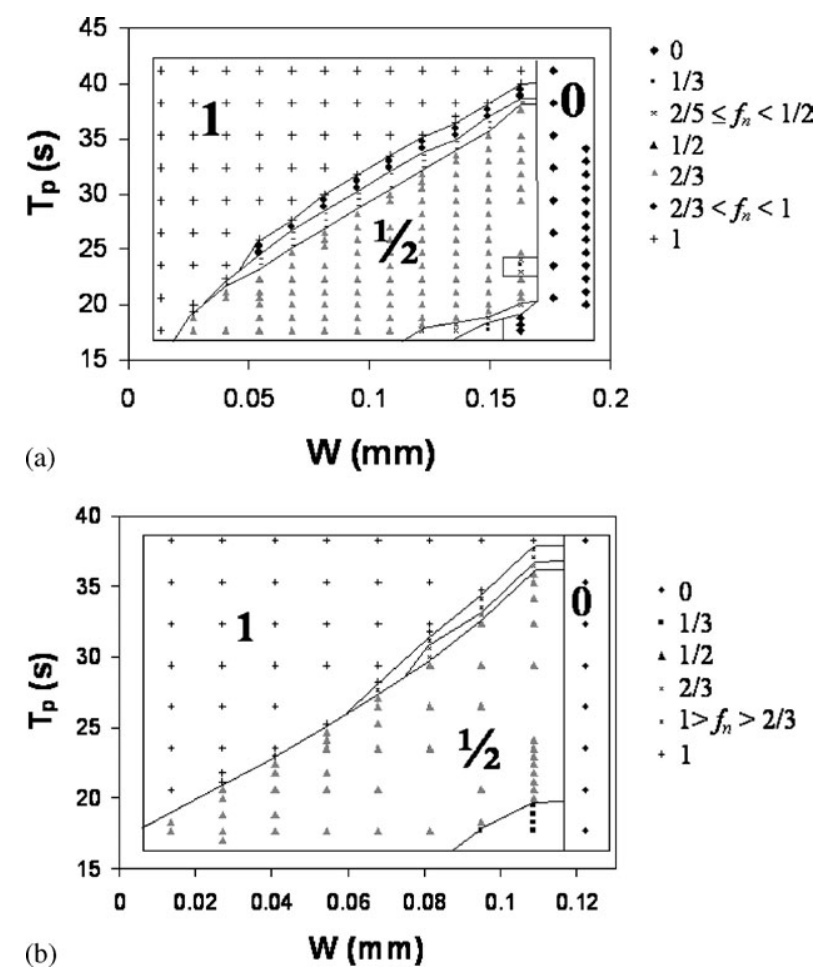

Fig. 1 The fraction of waves $f_{n}$ in a train of period $T_{\mathrm{p}}$ which cross the gap of width $W$ for $\varepsilon=0.054$ (a) $f=2.4$ and (b) $f=3$.

\section{Results}

\section{One-gap system}

A phase diagram is constructed for a system with one gap showing the fraction of waves in a wave train with period $T_{\mathrm{p}}$ crossing the domain as a function of the gap width $W$ (Fig. 1). In general, $f_{n}$ increases with increasing period and decreasing gap width. The critical gap width $W_{\text {cr }}$ decreases when the excitability is decreased: $W_{\mathrm{cr}}=0.163 \mathrm{~mm}$ for $f=2.4$ (Fig. 1(a)), and $0.110 \mathrm{~mm}$ for $f=3$ (Fig. 1(b)). A narrow band of complex behaviour separates the values of $T_{\mathrm{p}}$ and $W$ for which all waves pass $\left(f_{n}=1\right)$ and 1 in 2 waves pass $\left(f_{n}=1 / 2\right)$. This region of complex behaviour shrinks upon increasing $f$ to 3 (Fig. 1(b)).

A space-time plot shows 2 out of 3 waves successfully crossing the gap, i.e. the behaviour $f_{n}=2 / 3$ in Fig. 2(a). The light region corresponds to a high concentration of the oxidised form of the catalyst. We examined the trajectory in the $u-v$ phase plane for this behaviour. The concentrations of species $v$ and $u$ were recorded at the second excitable space point after
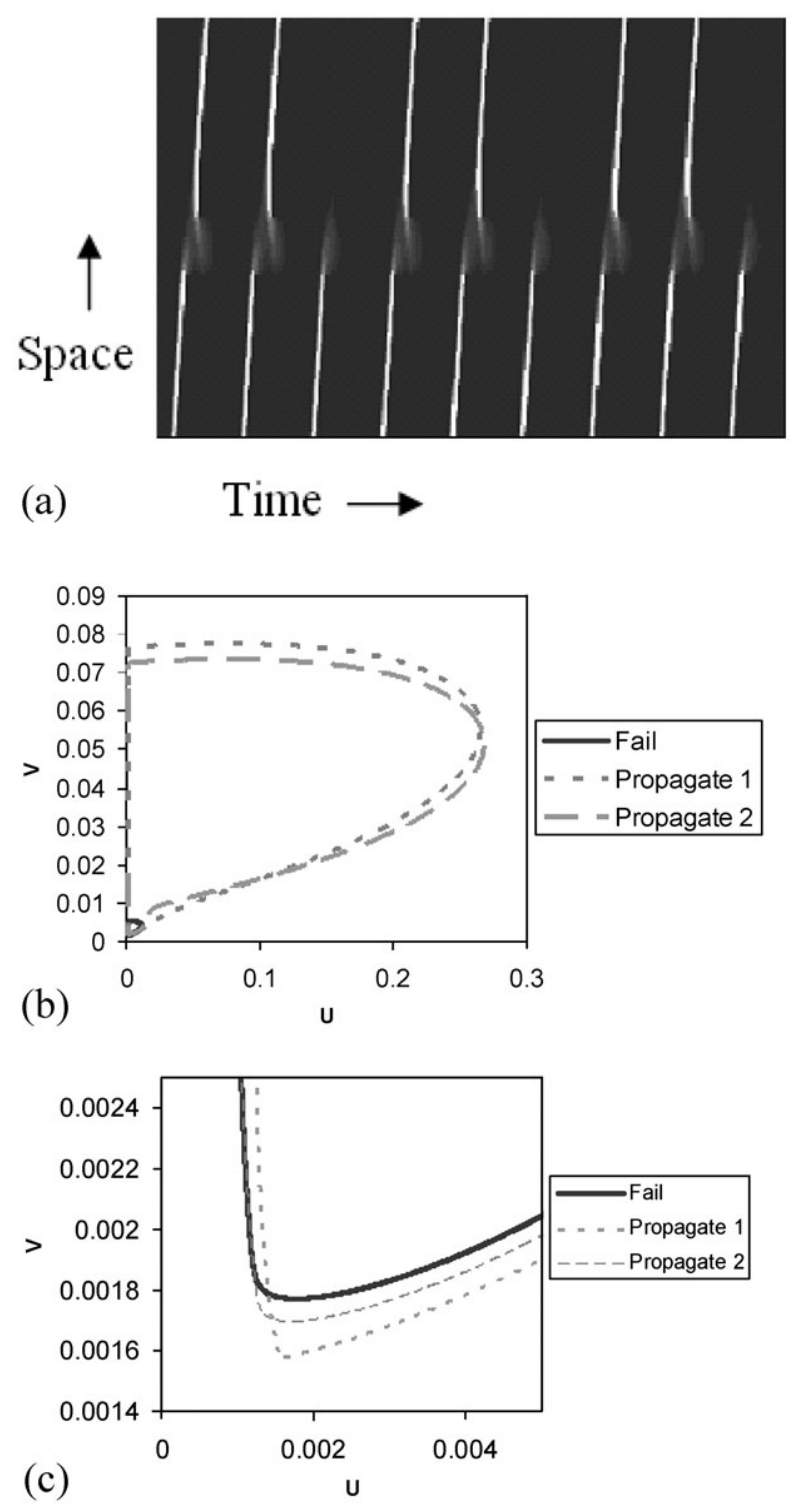

Fig. 2 (a) Space-time plot for a train of waves (light region) with period $T_{\mathrm{p}}=35.3 \mathrm{~s}$ crossing a gap of width $W=0.108 \mathrm{~mm}$, for a system $f=3$ and $\varepsilon=0.054$. (b) Trajectory in the $u-v$ phase plane, for three consecutive waves shown in Fig. 2(a). Concentrations of $v$ and $u$ determined at the second spatial point after the gap. The bottom left corner of (b) is magnified in (c). 


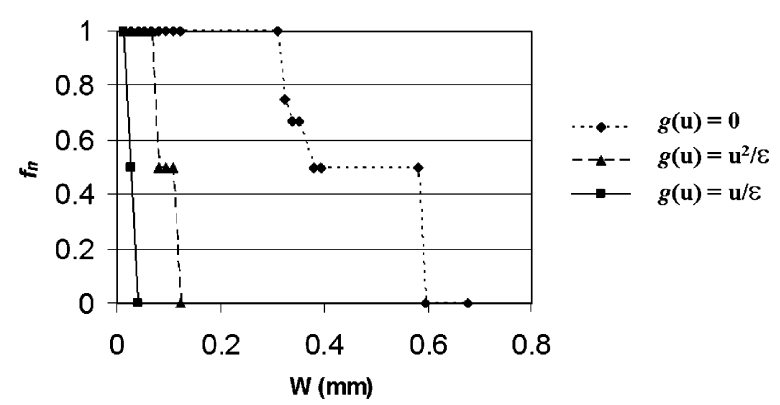

Fig. 3 Fraction of waves $f_{n}$ in a train of period $T_{\mathrm{p}}=29.4 \mathrm{~s}$ crossing a gap of width $W$ for a system with $f=3, \varepsilon=0.054$, and varying gap kinetics: $g(u)=0, g(u)=u / \varepsilon$ and $g(u)=u^{2} / \varepsilon$.

the gap and plotted for three consecutive waves (Fig. 2(b)). A macroscopic growth in $u$ (and hence a propagating wave) occurs following a perturbation in this species as a result of the diffusion of a supercritical amount of $u$ across the gap. After the passage of the first wave (propagate 1), the perturbation in $u$ occurs at successively higher values of $v$, such that the second perturbation initiates a wave (propagate 2), but the third does not.

The effect of activator gap kinetics on the passage of the wave across the gap was investigated through variation of $g(u)$. We determined the fraction of waves in a train $\left(T_{\mathrm{p}}=29.4 \mathrm{~s}\right)$ crossing as a function of gap width (Fig. 3) for different kinetic orders. For each of the three cases, the concentration profile of the activator species is shown at successive time intervals in Fig. 4. The dotted vertical line represents the gap. The waves cross the gap when $g(u)=0$ and $g(u)=u^{2} / \varepsilon$ in Fig. 4(a) and 4(c), but wave block occurs when the activator decay within the gap is first order, $g(u)=u / \varepsilon$, as seen in Fig. 4(b).

\section{Two-gap system}

For an excitable domain containing two gaps, we determined the fraction of waves $f_{n}$ passing across the gaps as a function of the spacing between the gaps $S$ (Fig. 5). The critical spacing is that for which waves crossing gaps separated by a smaller space will fail. The value of $S_{\text {crit }}$ increases with increasing gap width from $S_{\text {crit }}=0.014 \mathrm{~mm}$ for $W=0.068 \mathrm{~mm}$, to $S_{\text {crit }}=$ $0.054 \mathrm{~mm}$ for $W=0.108 \mathrm{~mm}$.

Frequency transformation was observed at both the first and second gaps. We define failure at gap 1 if no growth of the activator species is observed in the space between gaps and failure at the second gap if growth is observed, but no wave is initiated on the other side of gap 2. For example, with $W=$ $0.095 \mathrm{~mm}, T_{\mathrm{p}}=35.3 \mathrm{~s}, S=0.027 \mathrm{~mm}, f_{n}=0$, failure occurs at the second gap (Fig. 6(a)). Interestingly, we also found that in some cases, the fraction of waves successfully passing both gaps decreased upon increasing the spacing between gaps: for the same conditions as Fig. 6(a), but with $S=0.041 \mathrm{~mm} ; f_{n}=$ $2 / 3$ (Fig. 6(b)), and with $S=0.054 \mathrm{~mm} ; f_{n}=1 / 2$ (Fig. 6(c)). Frequency transformation occurs at gap 1 in the latter case, and corresponds with a larger growth of $u$ in the space between gaps.

\section{Multiple-gap system}

For a multiple gap system we define $f_{n}$ as the fraction of waves which cross the entire domain, for systems consisting of 1,2 , etc., up to 7 gaps. The fraction $f_{n}$ is plotted versus number of gaps in the system, for different values of $f$ and $\varepsilon$ (Fig. 7). Depending on the excitability, $f_{n}$ is unaffected by the number of gaps (high excitability) or decreases with the number of gaps (reduced excitability). The fraction $f_{n}$ is extremely sensitive to the values of $f$ and $\varepsilon$ : increasing $f$ from 3.00 to 3.01 results in waves unable to cross a system consisting of more than three
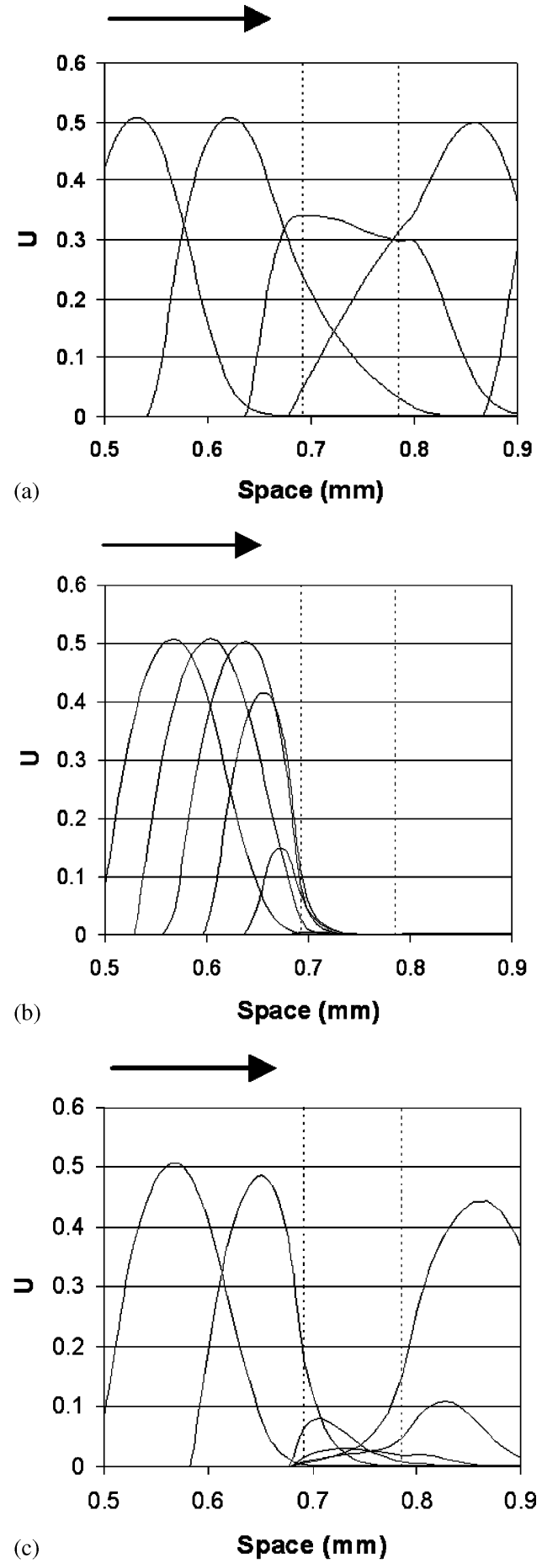

Fig. 4 Successive activator concentration profiles for a train of waves $\left(T_{\mathrm{p}}=29.4 \mathrm{~s}\right)$ crossing a gap of width $W=0.095 \mathrm{~mm}$ for a system with $f=3, \varepsilon=0.054$, and varying gap kinetics: (a) $g(u)=0$, (b) $g(u)=u / \varepsilon$ and (c) $g(u)=u^{2} / \varepsilon$. The arrows indicate the direction in which the waves are travelling and the time difference between curves is $0.25 \mathrm{~s}$ for (a) and (c) and $0.1 \mathrm{~s}$ for (b).

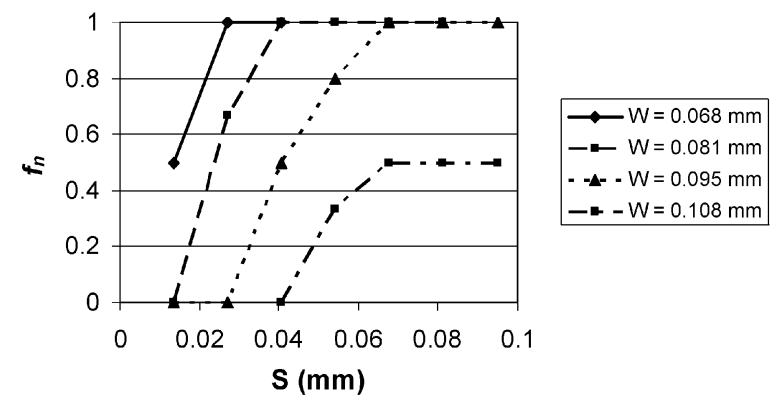

Fig. 5 The fraction of waves crossing two gaps as a function of the spacing between gaps for various gap widths. 


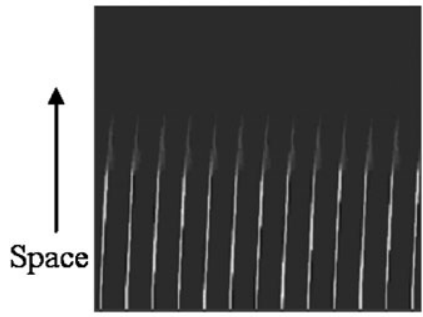

(a) Time

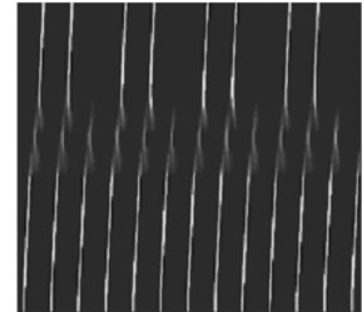

(b)

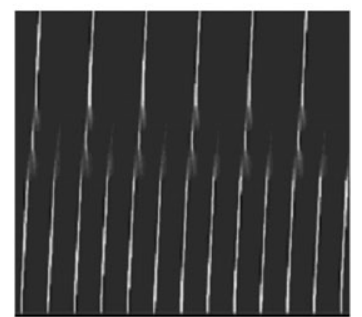

(c)

Fig. 6 Space-time plot for two-gap system with $W=0.095 \mathrm{~mm}, T_{\mathrm{p}}=35.3 \mathrm{~s}, f=3, \varepsilon=0.054$ and (a) $S=0.027 \mathrm{~mm}$ (b) $S=0.041 \mathrm{~mm}$, (c) $S=0.054 \mathrm{~mm}$.
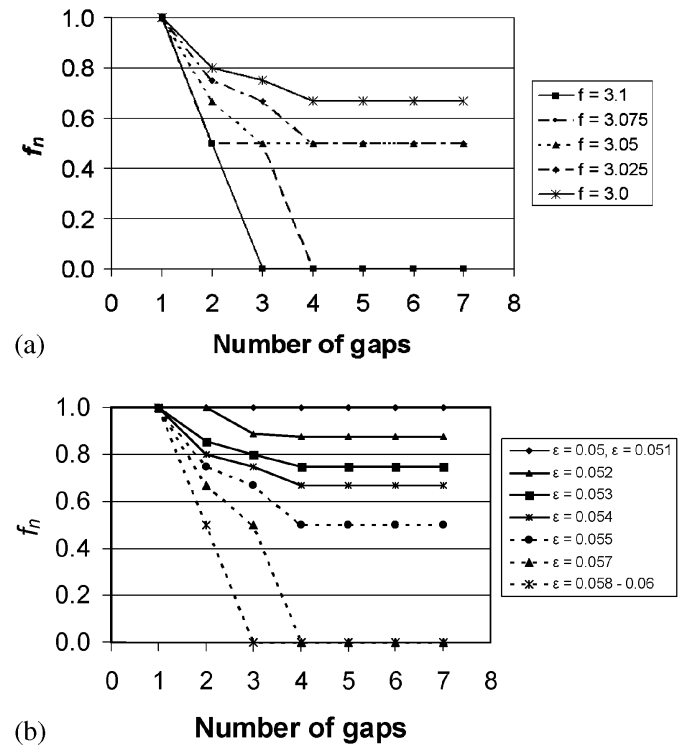

Fig. 7 Fraction of waves crossing the entire domain as a function of number of gaps in the system, for $W=0.095 \mathrm{~mm}, S=0.054 \mathrm{~mm}$, $T_{\mathrm{p}}=35.3 \mathrm{~s}$ and various values of $f$ and $\varepsilon$.

gaps. Space-time plots are constructed for two scenarios: number of gaps $=3, f_{n}=1 / 2$ and number of gaps $=7, f_{n}=0$ (Fig. 8). We examined profiles of the activator $u$ in the seven-gap system $\left(f_{n}=1\right)$ and found that the maximum concentration of $u$ in the space between gaps decreases with increasing number of gaps (Fig. 9).

\section{Discussion and conclusions}

Periodic perturbation of excitable media is an important area of study due to its connection with cardiac arrhythmias. High frequency periodic forcing of the atrioventricular (AV) node can lead to a 'dropped beat' i.e. not all periodic stimuli lead to ventricular contraction. These "Wenckebach rhythms" can be explained by the existence of a refractory period i.e. a time during which a second stimulus cannot lead to excitation. ${ }^{5}$ Heterogeneity in the excitable medium may contribute to wave break-up and associated potentially fatal arrhythmias. ${ }^{6}$ It has been demonstrated that physical obstacles, caused by cell death within the heart, can lead to wave block and if an excitation wave propagates through an excitable medium with local differences in recovery times, wave break-up can occur. ${ }^{7,19}$ In this paper we further investigate the periodic perturbation of a heterogeneous excitable medium, which consists of excitable regions separated by "gaps". If any gap in the array is bigger than a critical value $W_{\text {crit }}$, then the gap acts as anstacle and the wave is blocked. The value of $W_{\text {crit }}$ predicted in simulations (ranging between 0.110 and $0.163 \mathrm{~mm}$, see Fig. 1) agrees well with that observed in experiments: $0.13 \mathrm{~mm}^{8}$

The frequency transformation $\left(f_{n}\right)$ of a train of waves crossing a gap can be explained by considering the medium as a periodically forced excitable system ${ }^{14,20,21}$ where the diffusing activator perturbs the excitable medium after the gap. There is a shift in the phase of oscillation when the medium is perturbed by an incoming wave. The phase $\varphi$ is defined $t / T_{\mathrm{B}}$ where $t$ is the time required to move to some point of the oscillatory cycle and is between 0 and $T_{\mathrm{B}}$ and $T_{\mathrm{B}}$ is the refractory time of the excitable system, therefore: $0 \leq \varphi \leq 1$. This shift is characterised by plotting the new phase, post perturbation, against

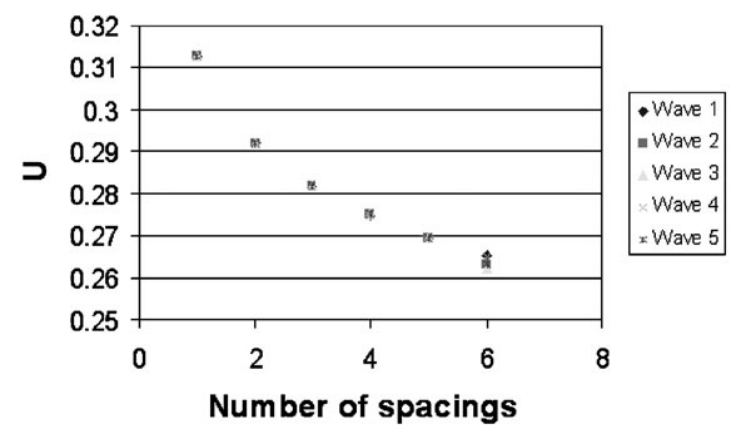

Fig. 9 Maximum value of $u$ in the space between gaps, for a 7 gap system with $f_{n}=2 / 3$.
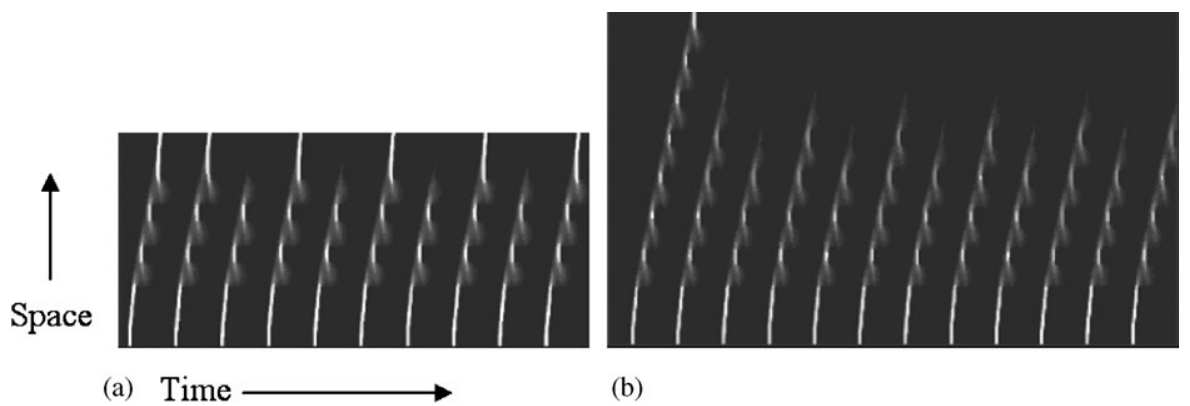

(b)

Fig. 8 Space-time plots for multiple gap systems with $W=0.095 \mathrm{~mm}, S=0.054 \mathrm{~mm} T_{\mathrm{p}}=35.3 \mathrm{~s}, f=3, \varepsilon=0.056$ (a) number of gaps $=3 ; f_{n}=1 / 2$ (b) number of gaps $=7 ; f_{n}=0$. 
the old phase to give the phase-excitation curve (PEC). Marek and co-workers ${ }^{20,22,23}$ developed one-dimensional maps based on PEC's to describe periodically forced systems. This was further developed by Tóth et al. ${ }^{14}$ using the dimensionless forcing period $\tau_{\mathrm{f}}$, defined as $T_{\mathrm{f}} / T_{\mathrm{B}}$ where $T_{\mathrm{f}}$ is the forcing period in seconds and in our case is the period of the wave train. The values of $f_{n}=1,1 / 2$ and 0 are seen over a large range of $\tau_{\mathrm{f}}$ and the more complex patterns are seen over smaller ranges. Our results agree with this interpretation.

In agreement with experiments, ${ }^{8}$ the fraction of waves $f_{n}$ crossing the domain depends on the excitability of the medium (through the initial concentrations), the period between initiations, the number and width of gaps, and the size of the spacing between gaps. Decreasing the period and the excitability of the system results in a higher super-critical threshold of $u$ required for the initiation of a wave on the other side of a gap, and hence $f_{n}$ decreases. This "dynamic threshold" depends greatly on the diffusion constant of the activator species $u$, and will differ from that of the well-stirred system. ${ }^{14,24}$ The values of the gap width $W$ and spacing $S$ affect the actual amount of $u$ which reaches the other side of the gap. In general, $f_{n}$ decreases with increasing $W$ or decreasing $S$ as these result in a lower amount of $u$ reaching the other side of the gap. The surprising result that under certain conditions a bigger value of $S$ reduces the fraction of waves crossing the domain might also be explained within this framework: a larger $S$ in this case results in a larger growth of $u$ and a longer recovery period, thereby increasing the threshold amount required to initiate a second wave. We also find a maximum number of gaps that a wave can propagate across, but this is very sensitive to initial conditions. At critical values of $f$ and $\varepsilon$ we observe the maximum number of gaps to be 3 and 4 respectively.

The motivation behind studying various gap kinetics was to ensure that the resonance effects are not specific to the second order rate law assumed from the FKN model. Although the parameter ranges for resonance effects and the specific value for the critical gap width change quantitatively with the reaction order, the generic qualitative features are preserved for other rate laws. With first order removal, and assuming a dimensionless rate coefficient still determined by the parameter $\varepsilon$ for ease of comparison, the critical gap width is decreased compared with the second order form, consistent with a faster "washing-out" effect. The existence of a critical width and of resonance effects even persists in the absence of kinetic loss in the passive gap: in this case, the critical gap width is approximately five times larger than with second order loss, and the range of $W$ for which frequency transformations were observed also increases dramatically.

Finally, we were able to observe numerically a "weakening" effect following the passage of the wave over multiple gaps. Thus if the wave corresponded to a re-entrant structure, for example if the passage of the wave was around a 1-d ring, then even in cases where the heterogeneities in the excitable medium were small, the wave would eventually die out. Whether such an effect might contribute to wave break-up in 2-d scenarios will be the subject of further investigations.

\section{Acknowledgements}

GRA and VG would like to thank ESF REACTOR Programme for support and AFT would like to thank the Nuffield foundation (NUF-NAL) and the EPSRC (GR/509753/01) for financial support. VG also thanks OTKA (T038071) for financial support.

\section{References}

1 A. F. Taylor, Prog. React. Kinet., 2002, 27, 247-325.

2 J. P. Keener, SIAM J. Appl. Math., 2000, 61, 317-334.

3 J. Sneyd and J. Sherratt, SIAM J. Appl. Math., 1997, 57, 73-94.

4 A. T. Winfree, When Time Breaks Down, Princeton University Press, Princeton, 1987.

5 L. Glass and M. C. Mackey, From Clocks to Chaos, Princeton University Press, Princeton, 1988.

6 G. Bub, A. Shrier and L. Glass, Phys. Rev. Lett., 2002, 88, 058101.

7 G. Bub and A. Shrier, Chaos, 2002, 12, 747-753.

8 A. F. Taylor, G. R. Armstrong, N. Goodchild and S. K. Scott, Phys. Chem. Chem. Phys., 2003, 5, 3928-3932.

9 K. Suzuki, T. Yoshinobu and H. Iwasaki, J. Phys. Chem. A, 2000, 104, 5154-5159.

10 K. Agladze, R. R. Aliev, T. Yamaguchi and K. Yoshikawa, J. Phys. Chem., 1996, 100, 13895-13897.

11 T. J. Lewis and J. P. Keener, SIAM J. Appl. Math., 2000, 61, 293-316.

12 J. Sielewiesiuk and J. Gorecki, Phys. Chem. Chem. Phys., 2002, 4, 1326-1333.

13 J. Sielewiesiuk and J. Gorecki, J. Phys. Chem. A, 2002, 106, 4068-4076.

14 Á. Tóth, V. Gáspár and K. Showalter, J. Phys. Chem., 1994, 98, 522-531.

15 R. J. Field and R. M. Noyes, J. Am. Chem. Soc., 1974, 96, 2001-2006.

16 R. J. Field, E. Körös and R. M. Noyes, J. Am. Chem. Soc., 1972, 94, 8649-8664

17 J. J. Tyson and P. C. Fife, J. Chem. Phys, 1980, 73, 2224-2237.

18 http://www.math.pitt.edu/ bard/xpp/xpp.html.

19 J. M. Starobin, Y. I. Zilberter and E. M. Rusnak, Biophys. J., 1996, 70, 581-594.

20 J. Finkeová, M. Dolnik, B. Hrudka and M. Marek, J. Phys. Chem., 1990, 94, 4110-4115.

21 J. Sielewiesiuk and J. Górecki, Phys. Rev. E., 2002, 66, 016212.

22 M. Dolnik and M. Marek, J. Phys. Chem., 1991, 95, 7267-7272.

23 M. Dolnik, J. Finkeova, I. Schreiber and M. Marek, J. Phys. Chem., 1989, 93, 2764-2774.

24 H. Sevcikova and M. Marek, Physica D, 1991, 49, 114-124. 